\title{
IDENTIFICATION OF FOOD INSECURE ZONES USING REMOTE SENSING AND ARTIFICIAL INTELLIGENCE TECHNIQUES
}

\author{
K. Nivedita Priyadarshini ${ }^{1, *}$, Minakshi Kumar ${ }^{2}$, K. Kumaraswamy ${ }^{1}$ \\ ${ }^{1}$ Dept. of Geography, Bharathidasan University, Tamil Nadu, India - nivi.darshini@yahoo.com, kkumargeo@gmail.com \\ ${ }^{2}$ Indian Institute of Remote Sensing (ISRO), Uttarakhand, India - minakshi@iirs.gov.in \\ Commission V, SS: Emerging Trends in Remote Sensing
}

KEY WORDS: Food insecure zones, food insecurity, artificial intelligence, neural network, hunger index score, risk metric

\begin{abstract}
:
The basic human need is to ensure adequate access to food without any combat, loss of productivity and cognitive impairment in the supply chain. When an individual is limited to proper procurement of food through various determinants there stems sustained hunger which is termed as 'food insecurity'. The study portrays to identify the food insecure zones using indicators which are implemented methodically through remote sensing and artificial intelligence techniques. Madhya Pradesh being a semi arid region faces reduction in the agro ecosystem due to the climatic changes and rainfall impacts which are the key trends for demand of food and production thus resulting in risk of malnutrition and hunger. Tackling food shortage requires addressing both environmental and socio-demographic factors in order to minimize food insecurity. The spatial variation of rainfall over years along with significant land degradation affects the common cultivation pattern among the households. In this study, a neural network approach is employed to identify the zones that ensure less access to food using indicators which mainly focuses on child population below five years, hunger index measuring parameters like child stunted, child wasted, children undernourished, child mortality below five years along with supporting environmental factors such as land use/land cover, NDVI and rainfall prevailing in the study area. The result shows a bleak statistics of villages representing the hunger index score that are categorized into low, serious, alarming and extremely alarming estimating a count of 70, 73, 23 and 7 villages respectively in the entire study area.
\end{abstract}

\section{INTRODUCTION}

\subsection{Hunger - An illustration}

The people suffering from hunger we usually refer to those who, for sustained periods, are unable to eat sufficient food to meet basic nutritional needs. i.e. for weeks, even months, its victims must live on significantly less than the recommended nutritional levels that the average person needs to lead a healthy life. In modern times, hunger is not the product of a lack of food rather; hunger mainly occurs due to food distribution problems. The poverty hunger nexus is considered the most important factor in causing hunger among people. The poverty-stricken do not have enough money to buy or produce enough food for themselves and their families. Hence, poor becomes hungry and hunger traps them in poverty (Kumar, 2017).

\subsection{Global Hunger Index vs. India Hunger Index}

International Food Policy Research Institute prepares an annual report on the level of hunger globally which portrays the inequalities of hunger through the Global Hunger Index score for each country. The GHI scores are based on the multidimensional parameters of hunger. The level of hunger had been decreased by 27 percent. Of the 119 countries assessed in 2017 year's report, one falls in the extremely alarming range on the GHI Severity Scale; 7 fall in the alarming range; 44 in the serious range; and 24 in the moderate range (IFPRI, 2017). With more than 200 million food-insecure people India is home to the largest number of hungry people in the world (FAO, 2008).

* Corresponding author
In 2017 GHI, India scored 31.4 and ranked $100^{\text {th }}$ among 119 countries was placed in high end of 'serious' category. The proportion of undernourished in population was $14.5 \%$, prevalence of wasting in children under five years was $21.0 \%$, prevalence of stunting in children under five years was $38.4 \%$ and under 5 mortality rate was $4.8 \%$. Despite India being the second largest food producer, it has the second highest population of undernourished population.

\subsection{Continuing quest for food security in Madhya Pradesh}

The main objective of the development of an India state hunger index is to focus on the problem of hunger and malnutrition at the state and central levels in India (Saxena). Madhya Pradesh is the most affected state in India among the 17 states of calculated index (India State Hunger Index, 2009). The state level hunger index was calculated over 95 percent of total population with respect to the Census of India. The India state hunger index revealed the severity of hunger across the states where most states had 'serious' hunger problem while Madhya Pradesh was the only state under 'extremely alarming' hunger problem.

The Indian state hunger index score of Madhya Pradesh was 30.87 and it ranked $17^{\text {th }}$. It was surveyed and reported that states having number of rural residents who take less than 2 meals a day have adverse food insecurity (UNDP, 2007). Also poverty and hunger persists among the people of Madhya Pradesh where there is lack of proper public distribution system. It is stated that, families affected by chronic hunger in villages of most part of Madhya Pradesh have to live with hunger for around 4 months of the year (Jain, 2011). Using even an inflated count, they get ration sufficient for no more than 8 months of the year. 
This paper, aims to propose the Regional level hunger index for the villages in the study area and categorizing food insecure zones such as 'low', 'serious', 'alarming' and 'extremely alarming' based on risk metric. It is achieved by constructing a perceptron using custom neural network tool in MATLAB. This uses many raw determinants which are both significant and supportive that is trained tested and validated.

\section{RELATED WORKS}

After the late 1950s, the considerable growth of interest concerningthe resolving of problems facedin the image processing(Andrews, 1970; Rosenfeld, 1969), pattern recognition ( $\mathrm{Fu}, 1974$; Tou and Gonzalez, 1974) and scene analysis (Winston, 1975), have developed many experimental softwares and theoretical methods to enhance the accuracy of image processing systems(Fu and Rosenfeld, 1976; Dean et al., 1995).The classical image processing systems makes use of the existing images in the production of the new images, which are classified in atwo-dimensional interpertation techniques of three-dimensional scenes to determine the nearest object classification and its assosciated confidence level using a local statistical analysis.

Due to whichvarious artificial intelligence techniques of image processesing were emerged as a major threshold area in response to the ignorance of symbolic information dealing with contextual realtionships, object attributes and physical constraints in the classical image processing technology (Gilmore, 1985).Thus, the main component of image processesing through artificial intelligence is that the computer based systems have a complete understanding of the physics of image formation through exploiting the internal symbolic representation of the scene by determining the measurements of the input image using the lighting, shape and surface material of the objests being imaged (Horn, 1979).The high-level human capabilities were mimiced by constructing and building algorithmsusing first type of classical artificial intelligence such as frame based, rule based and case based reasoning for expert systems, logical and probabilistic inference, nonmonotonic reasoning etc (Crabbe and Dubey, 2000).

The problem arised with the classical artificial intelligence was that it was deductive and assumption-based rather than inductive and problem-based. In order to solve this issue the machines were trained with a algorithm so that it can learn from the examples of training algorithms (Mitchell, 1997).One among the training algorithm based tools was artificial neural networks.The artificial neural networkswere developed in the idea of modelling a human brain were a large number of neuron models resemple interconnected neurons as same as in our human brain from which researchers understood that this system of neural networks can possibly perform any kind of complex computations (McCulloch and Pitts, 1943). Later on, many modifications and development of single layer perceptrons (Nilsson, 1965) and multi-layer perceptrons were carried out in the studies which was conducted between 1950's and 1980's.Multi-layer perceptrons also called as feed-forward artificial neural networks, self-organising maps (SOMs) and Hopfield neural networks (HNN) and etc. Various studies employs different kinds of neural networks. Back Propagation Neural Network (BPNN) algorithm was employed for the classification of features such as urban/ rural/ deserted areas from input satellite imagery using mean shift clustering with artificial neural network in which images were classified by grouping pixels belonging to rural, urban neither rural nor urban (Sharma et al., 2016). The classification of urban aerial data based on pixel labelling with deep convolutional neural networks and logistic regression is adapted to the high resolution aerial images for feature extraction and classification (Yao et al., 2016).

Using the above mentioned references, a study had been carried out using the neural networking technique to identify the 'Food insecure zones'. The layers and databases were taken as input neurons in order to detremine the output that are processed in the hidden layer using custom equation. This research fulfils two objectives in which first concentrates on training raw input data and second focuses on calculation of regional hunger index for the study area chosen. This research produces productive results that could further be hosted for the use of public updation if essential.

\section{STUDY AREA AND DATASETS}

\subsection{Study area description}

Niwas is a tehsil in Mandla District of Madhya Pradesh State, which is the geographical heart of India. It belongs to Jabalpur Division. The extent of the tehsil is $23^{\circ} 2^{\prime} 8.0545^{\prime \prime} \mathrm{N}$ latitude and $80^{\circ} 26^{\prime} 16.048^{\prime \prime} \mathrm{E}$ longitude. The total area coverage is 2020.29 Sq.Km. There are approximately 173 villages in the tehsil. As per Census 2011 out of total population, 6.6\% people lives in urban areas while $93.4 \%$ lives in the rural areas. Also the sex ratio of urban areas in Niwas tehsil is 971 while that of rural areas is 1,023 . The population of children between ages 0 6 years in Niwas Tehsil is 18198 which are $15 \%$ of the total population. There are 9187 male children and 9011 female children between the ages $0-6$ years. This district is completely covered by forest and hills with dominated tribal population. The study area has a subtropical climate which has hot dry summer and cool dry winters. The average rainfall is $137 \mathrm{~cm}$ and it decreases from east to west. The altitude of Niwas tehsil is 416 meters above mean sea level. The predominant type of soil in the study area is Barra which covers $57 \%$. This kind of soil is comprised of very poor qualities of black soil, stony or underlying rock materials that are not suitable for cultivation of any crop. The economy of the people living in these villages is by rearing cattle and livestock. Some move to the urban circles seeking labour for supporting livelihood.

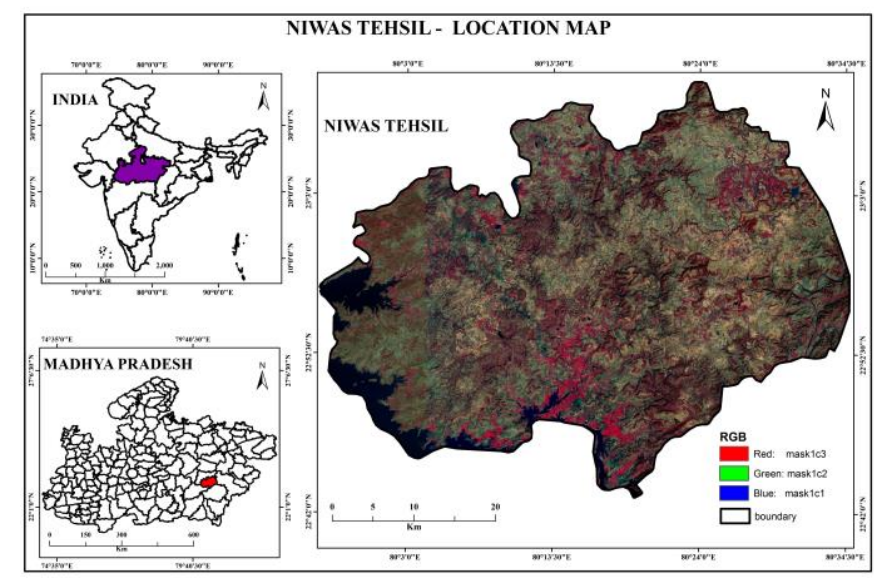

Figure 1. Niwas tehsil - Location map 


\subsection{Data Used}

A list of datasets had been used as training samples to maintain the performance of the neural network using MATLAB R2016a software. The remote sensor data of Sentinel 2 (bands of $10 \mathrm{~m}$ resolution) were stacked and samples from each class were trained to obtain landuse/landcover and NDVI layers using neural network algorithms that are discussed in methodology flowchart. Rainfall level that prevailed in the study was obtained from INSAT 3D IMR (Insat Multispectral Rainfall), a 6 channel imagery of $1 \mathrm{~km} * 1 \mathrm{~km}$ resolution. Census data 2011 were collected which mainly focuses on the total number of children present in each village in the whole tehsil. As this study aims at representing Regional level hunger, based on the dimensions of hunger given by the GHI, child population was given more preference. The hunger index parameters such as proportion of children undernourished (PUN \%), prevalence of child stunted under five years (CST \%), prevalence of child wasted under five years (CWA \%) and child mortality under five years $(\mathrm{CM} \%)$ were obtained from the National Family Health Survey (NFHS - 4) of the year 2015-16 and IFPRI 2017. The determinants like hunger parameters and population favours the study maximum and are termed as 'significant data' and other environmental factors like landuse/landcover, NDVI, rainfall that support the study are the 'supportive data'.

\section{METHODOLOGY}

\subsection{Workflow}

The overall workflow in Figure 2, shows the determinants that are trained using various algorithms and given as input to the custom neural network. Each of the layers and databases are trained tested and validated using the neural network algorithms which are processed and fed into the Multi Layer Perceptron (MLP) to obtain the regional level hunger index for a total of 173 villages present in Niwas tehsil.



Figure 2. Overall workflow

A schematic diagram of the input layer and databases are given below in Figure 3. The layers include raw remote sensor data containing pixel values which were segregated into five landuse/landcover classes, samples from each class were trained using algorithms discussed in 3.2. Databases were imported from various sources with suitable target datasets which were processed using nntool function in order to obtain the output database. Further, the output was analysed and a risk metric was created to categorize the villages based on the severity of hunger.

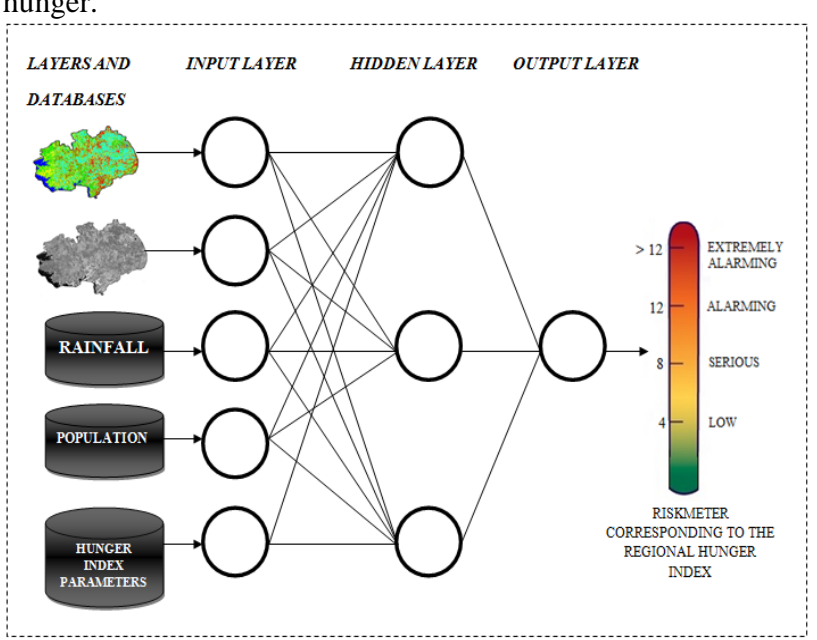

Figure 3. Network Architecture

\subsection{Neural Network algorithm}

Neural Network Toolbox provides algorithms, pretrained models, and apps to create, train, visualize, and simulate both shallow and deep neural networks (Mathworks, R2018a). Some of the tools of neural network application include:

- Pattern recognition tool - to recognize the difference between Normalized Differential Vegetation Index values shown in Figure 4, ranging between -1 to +1 were trained using DN values (Mathworks, R2017a).

- Cluster tool - an unsupervised learning method to assign the number of classes shown in Figure 5 and 6 , that are to be aggregated or clustered for landuse/landcover classification were trained using SOM (Self Organising feature Map) (Mathworks, R2017b). The dimensions of the raw data were $20976 * 10980$ and each of the training samples was given in a $3 * 3$ matrix. The colour difference in Figure 5. indicates the distance between the sample points.

Time series tool - Rainfall that had occurred over a period of 32 years was trained using the nonlinear autoregressive network with exogenous inputs (NARX) shown in Figure 7. This is a recurrent dynamic network, with feedback connections enclosing several layers of the network (Mathworks, R2016a). The regression value $\mathrm{R}=$ 0.98585 is the measure of correlation between the input and output targets. Time series response in Figure 8, indicates time point during training, testing and validation.

Fitting tool - The Census data of 2011 mainly focusing on the child population was taken village wise shown in Figure 9. The regression value $\mathrm{R}=0.63773$ display network outputs from training, testing and validation. Also, the hunger index parameters such as the percentage of children undernourished (PUN \%), percentage of children wasted (CWA \%), percentage of children stunted (CST \%) and the percentage of child mortality under five years $(\mathrm{CM} \%)$ are trained with respect to the total child population in the study area to acquire the regional hunger index using fitting tool which helps to select data, create and train the network, 
and evaluate its performance using mean square error and regression analysis (Mathworks, R2016b).

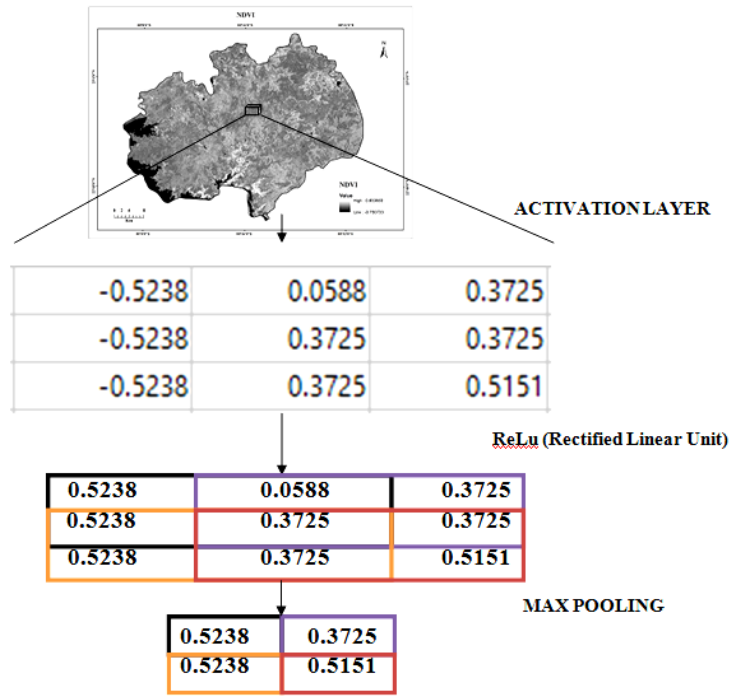

Figure 4. Image recognition mechanism

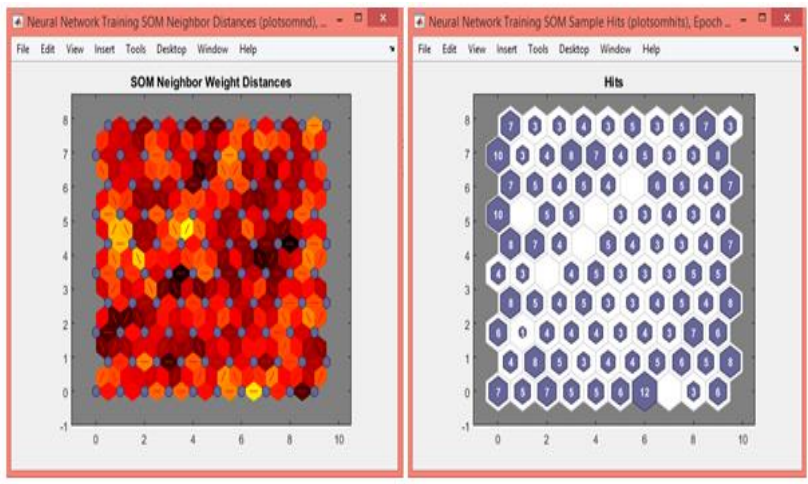

Figure 5. SOM neighbour weight distance Figure 6. Sample hits
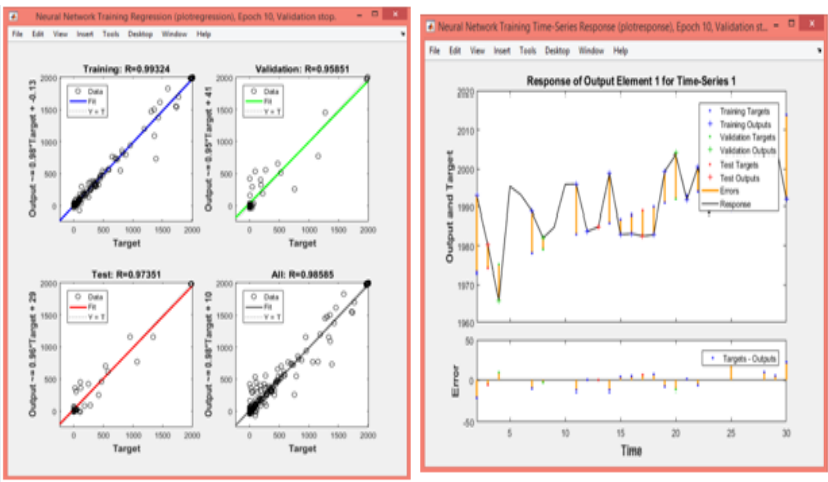

Figure 7. Time series regression

Figure 8. Time series response

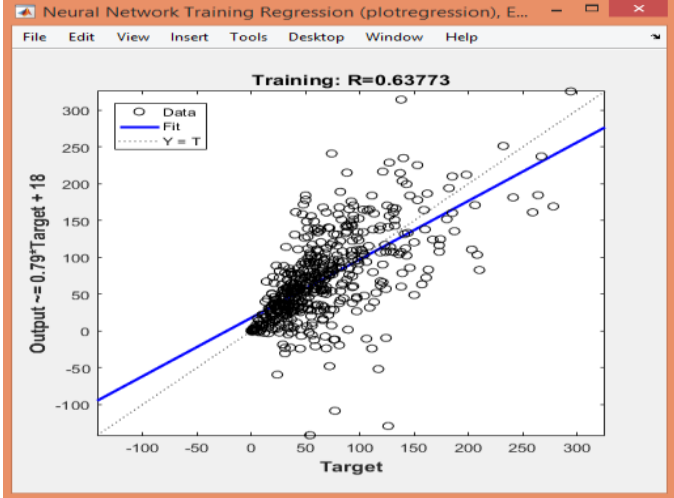

Figure 9. Fitting tool regression showing number of villages trained

\subsection{Custom Multi layer Perceptron network}

The neural network algorithm using the nntool is used to create a custom neural network in which the five trained neurons are given as input using a rule based mechanism determined in the custom equation 1 below,

Regional Hunger Index Score $=1 / 3\left[(\% \mathrm{PUN} / 80)^{*} 100\right]+$ $1 / 6[(\% \mathrm{CWA} / 30) * 100]+$ $1 / 6[(\% \mathrm{CST} / 70) * 100]+$ $\left.1 / 3[\% \mathrm{CM} / 35)^{*} 100\right]$

The network architecture shown in Figure 10, uses 5 trained input neurons to obtain the regional hunger index for 173 villages are processed using LEARNP learning function and HARDLIM transfer function.

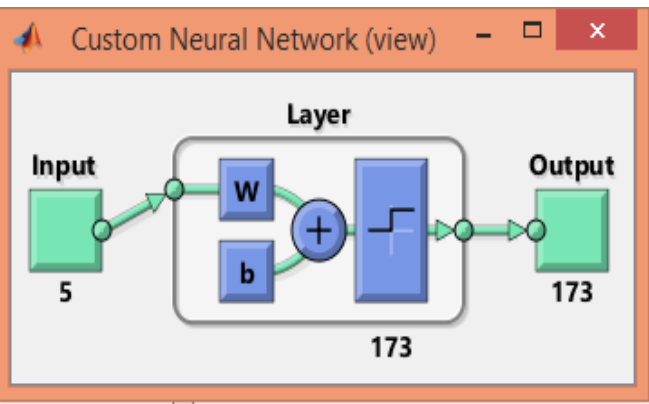

Figure 10. Custom neural network

\section{RESULTS AND DISCUSSION}

\subsection{Regional level hunger index estimation}

It is noticed that for whole of the Niwas tehsil the \% PUN52.4, \%CWA - 34.2, \% CST -39.4 and \%CM - 40.8. The custom equation provides the score for Niwas tehsil and it is found that the tehsil falls under 'extremely alarming' zone based on the State hunger index severity scale. Likewise, the regional hunger index score which is processed through the custom neural network is converted as a database and categorized into various food insecure zones based on risk metric created for 173 villages.

The regional level hunger index obtained is ranked into 4 categories based on the risk meter level such as, 


\begin{tabular}{|c|c|}
\hline RANK & RISK METER \\
\hline 4 & LOW \\
\hline 3 & SERIOUS \\
\hline 2 & ALARMING \\
\hline 1 & EXTREMELY ALARMING \\
\hline
\end{tabular}

Table 11. Ranking food insecure zones

\subsection{Analyzing and plotting}

The database is then imported into Classification Learner application which portrays the number of villages with respect to the regional hunger index score. It is found that out of 173 villages,

- $\quad 7$ villages - 'Extremely Alarming' Food insecure zone

- 23 villages - 'Alarming' Food insecure zone

- $\quad 73$ villages - 'Serious' Food insecure zone

- $\quad 70$ villages - 'Low' Food insecure zone

The analysed results are plotted in Google earth for verification. Further, the villages which fall under extremely alarming food insecurity could be suggested for immediate attention through the local NGO's.

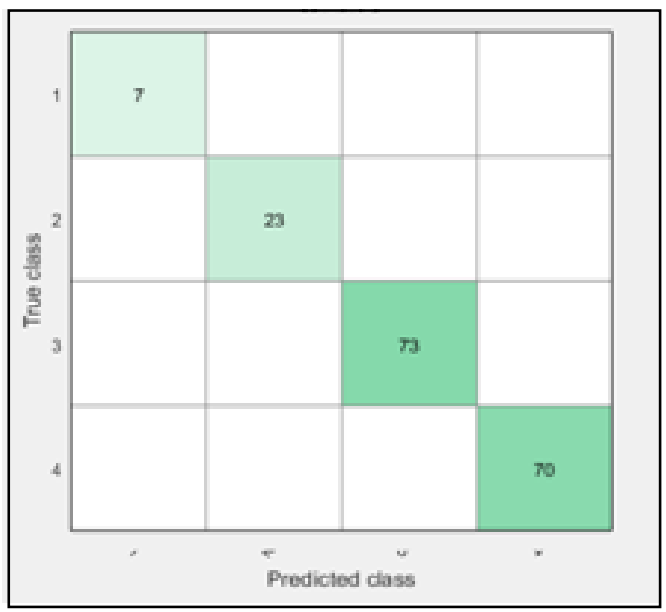

Figure 12. Matrix representing number of villages

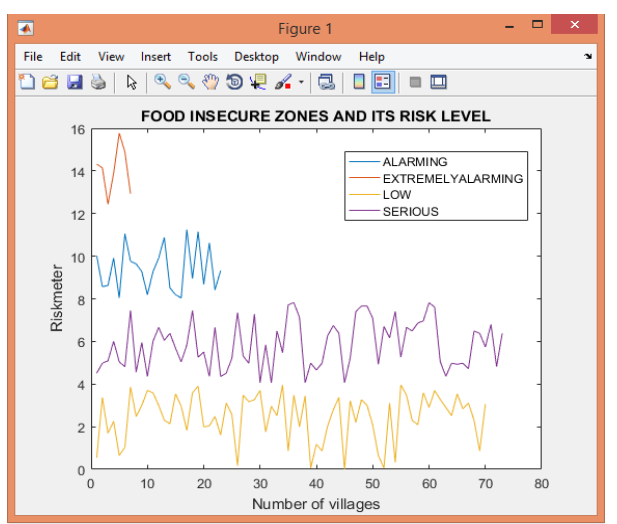

Figure 13. Number of villages with respect to risk metric

\subsection{Web map display using Geopoint}

A web map display using the generated output is established in MATLAB interface. The location details are plotted in the web map using Geopoint. A geopoint is a function for plotting the spatial data into a dynamic interface. Using geopoint, the details about the locations could be added and displayed in web map using a wmmarker tool.

The displayed result consists of information about the location, NGO organization near the village, its address and contact details using geopoint function.

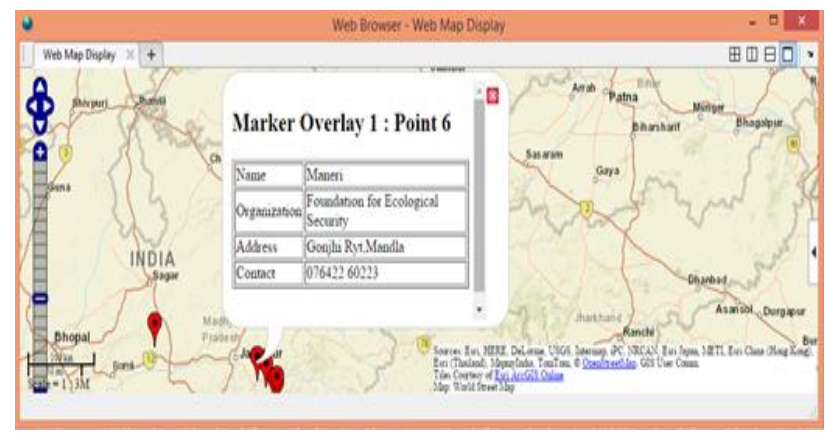

Figure 14. Webmarker display and adding geopoint



Figure 15. Spatial distribution of Regional hunger index

The villages falling under categories of food insecurity are thus represented spatially.

\section{CONCLUSION}

There are many such villages which lack proper procurement of food and basic needs in India as like Niwas tehsil, Madhya Pradesh. Though the supply of resources is abundant, there exists lack of proper distribution among the local divisions. In order to minimize the severity rate of hunger among the villages, a decision has to be taken by both the Government and other Non Governmental Organisations to work hand in hand for the health and well being of the people. Geospatial analysis using advancements like AI helps to spot zones that are at risk. 
Essential measures could be provided to the villages that are at an extremely alarming rate. Suitable agricultural business establishments among rural population must be initiated in order to increase yield in spite of poor climatic conditions. A climate smart agricultural system reduces the quest of hunger at a considerable rate. Awareness programs among the rural communities regarding the nutritional facts required for the growth of children below five years should be made mandatory. Food security in India can be achieved if there is proper attention given to rural areas which maintains sustainable health management practices for children below five years. This favours in eradicating hunger and make India a hunger free zone.

\section{REFERENCES}

Andrews, H. (1970). Computer Techniques in Image Processing. New York: Academic Press.

Dean, T., Allen, J., \& Aloimonos, J. (1995). Artificial

intelligence: Theory and practice.

FAO - The State of Food and Agriculture.(2008). Food and Agricultutal Organization.

Fu, K. (1974). Syntactic Methods in Pattern Recognition.

New York: Academic Press.

Fu, G., Liu, C., Zhou, R., Sun, T., \& Zhang, Q. (2017). Classification for High Resolution Remote Sensing Imagery Using a Fully Convolutional Network, 1-21. https://doi.org/10.3390/rs9050498

Gilmore, J. F. (1985). Artificial Intelligence In Image Processing. SPIE .

Horn, B. (1979). "Artificial Intelligence and the Science of Image Understanding, Computer Vision and sensor - based robots", Massachusetts: Plenum Publishing Corporation, pp. 65-77.

IFPRI. (2017). Global hunger index - The inequalities of Hunger.

India State Hunger Index. (2009). Comparisons of Hunger Across States, (February).

Jain, S. K. (2011). Valley of Food Insecurity and Chronic Hunger.

Kumar, G. R. (2017). Tackling Hunger in India. The Hans India.

McCulloch, W., \& Pitts, W. (1943). "A logical calculus of the ideas imminent in nervous activity", Bulletin of Mathematical Biophysics, 5, pp. 115-133.

Mitchell, T. (1997). Machine learning. McGraw Hill: New York.

Nilsson, N. (1965). Learning machines. New York: McGraw Hill.

Rosenfeld, A. (1969). Picture Processing by Computer. New York: Academic Press.
Saxena, N. C. (n.d.). CPRC. Retrieved from Hunger, Undernutrtion and Food security in India: https://assets.publishing.service.gov.uk/media/57a08ac540f0b6 52dd0008cc/CPRC-IIPA44.pdf

Sharma, A., Kaur, G., \& Gupta, N. (2016). ANN based GUI to Classify Satellite Images for Remote Sensing ANN based GUI to Classify Satellite Images for Remote Sensing, (September). https://doi.org/10.17577/IJERTV5IS090141

Tou, J., \& Gonzalez, R. (1974). Pattern Recognition Principles. United States: Addison-Wesley.

Human Development Report.(2007), United Nations Development Program(UNDP).

Winston, P. (Ed.). (1975). The Psychology of Computer Vision. New York: McCraw-Hill.

Yao, W and Krzystek, P. (2016). Classification of urban aerial data based on pixel labelling with Deep Convolutional Neural Network and Logistic Regression.

Website Reference:

https://in.mathworks.com/help/nnet/ug/train-and-applymultilayer-neural-networks.html- Retrieved from Mathworks. (R2018a).

https://in.mathworks.com/discovery/pattern-recognition.htmlRetrived from Mathworks. (R2017a).

https://in.mathworks.com/discovery/clusteranalysis.htmlRetrived from Mathworks. (R2017b).

https://in.mathworks.com/help/nnet/modeling-and-predictionwith-narx-and-time-delay-networks.htmlRetrived from Mathworks. (R2016a).

https://in.mathworks.com/help/curvefit/fit-comparison-in-curvefitting-app.html - Retrived from Mathworks. (R2016b). 